\title{
Etude Workforce, révision tarifaire et interprofessionnalité en ligne de mire
}

\section{Sandra Hügli-Jost \\ Responsable de communication mfe}

\section{C'est à nouveau à Lucerne et à nouveau dans le cadre du congrès du CMPR que s'est} tenue l'assemblée générale de mfe Médecins de famille et de l'enfance Suisse. Des températures très élevées à l'intérieur, le lac des Quatre-Cantons avec sa fraîcheur tentante pour de nombreux participants à l'extérieur et des moments inspirants lors du congrès, telles étaient les conditions dans lesquelles s'est déroulée l'assemblée générale de cette année.

Philippe Luchsinger, président de mfe Médecins de famille et de l'enfance Suisse depuis janvier 2017, a pour la première fois salué les membres de mfe. Conjointement avec les membres du Comité, il a présenté les activités de l'association au cours de l'année passée.

\section{mfe 2016}

\section{Comité}

A la fin 2016, mfe a pour la première fois depuis son existence vécu un changement de présidence. Marc Müller s'est retiré après 7 années passées à la présidence, mais il reste au sein du Comité en qualité de vice-président. Philippe Luchsinger a été élu nouveau président lors de l'assemblée des délégués, qui a eu lieu en automne. Ces deux personnages forment une équipe convaincante et le changement de président a ainsi pu se dérouler en toute fluidité.

\section{Politique}

En 2016, mfe a suivi des affaires politiques pertinentes, telles que la révision de la loi sur les produits thérapeutiques, la loi fédérale sur les professions de la santé (avec le crédit accordé à l'interprofessionnalité) ou l'augmentation du nombre de places d'études en médecine, qui est passé à 1300 . Ces dossiers ont été de la plus grande importance pour mfe et ont requis un engagement tout aussi conséquent. Le concept des médecins de premier recours, qui a été adopté en automne lors de l'assemblée des délégués, est activement mis en œuvre pour les entretiens et discussions avec les partenaires de dialogue pertinents. Sur cette base, nos points de vue et nos idées peuvent être communiqués de façon intelligible, homogène et claire.

\section{Tarifs}

En 2016 également, les travaux préparatoires relatifs à la révision tarifaire ont été extrêmement vastes et intensifs. La commission Tarif s'est activement engagée dans le processus de révision tarifaire et a défendu les points pertinents pour les médecins de famille et de l'enfance. Les membres de mfe ont suivi la recommandation de mfe concernant la votation générale de la FMH et ils ont rejeté la révision du tarif ambulatoire en 2016. La prise de position de mfe sur l'intervention tarifaire du Conseil fédéral a été déposée le 16 juin 2017, et les préoccupations des médecins de famille et de l'enfance ont ainsi été communiquées expressément. mfe poursuivra les discussions tarifaires sous l'égide de la FMH (processus Tarco) - l'objectif est et restera l'autonomie tarifaire basée sur le partenariat.

\section{Comptes/budget}

L'association mfe est parvenue à atteindre son objectif de fonds propres équivalent à un chiffre d'affaires annuel et à respecter son budget de dépenses. Une perte a néanmoins été enregistrée au niveau des recettes. Cette situation est due aux nombreux départs de l'association, ce qui s'explique en grande partie par l'envoi, pour la première fois, d'une facture de cotisation commune avec la SSMIG et certaines sociétés cantonales. La facture commune aurait normalement dû fournir un meilleur aperçu aux membres. Or, dans les faits, nous avons assisté à une vague de désistements, 


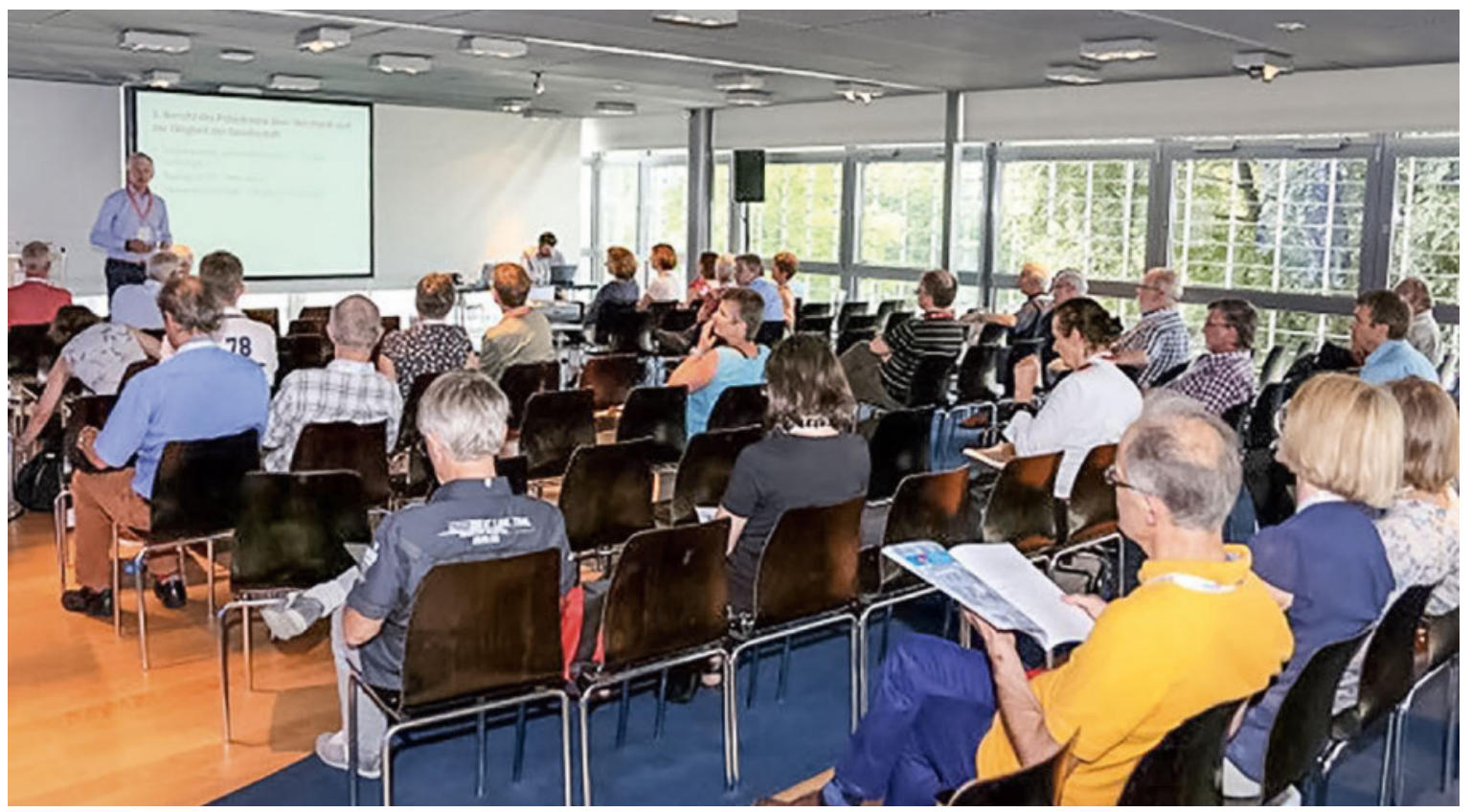

avec toutefois aussi un grand nombre de départs de membres retraités pour des raisons d'âge. A court et à moyen terme, mfe doit impérativement recruter de nouveaux membres afin de compenser la hausse prévisionnelle des départs liés à l'âge au cours des prochaines années.

\section{Interprofessionnalité}

L'interprofessionnalité reste une priorité stratégique de mfe. Dans ce contexte, la première formation ehpic de Suisse a été préparée conjointement avec une équipe canadienne de Toronto et elle s'est déroulée avec succès en mars 2017. Il est essentiel que mfe s'engage activement dans la formation interprofessionnelle, ce qui envoie des signaux majeurs à nos partenaires actifs dans le domaine des soins de premiers recours et de la politique.

\section{Secrétariat général}

En 2016, huit collaborateurs ont également apporté leur soutien à l'association mfe au niveau du secrétariat général décentralisé (Berne, Lichtensteig, Zurich et Lucerne). En plus de l'encadrement des sessions parlementaires, le secrétariat général a été occupé par diverses prises de position et procédures de consultation relatives à des affaires pertinentes. Outre la communication (modernisation du site internet, nouveau rapport annuel, travail médiatique, etc.), l'accompagnement continu des membres et le soutien du Comité et des délégués font également partie des activités quotidiennes.

\section{Etude Workforce}

L'étude Workforce, un projet commun de mfe et du Centre universitaire de médecine de premier recours des deux Bâle (Universitäres Zentrum für Hausarztmedizin beider Basel), a constitué une priorité en matière de communication. Les résultats de l'étude et les exigences politiques associées de mfe ont été communiqués avec succès à l'occasion d'une conférence de presse nationale. Dans ce cadre, la journée de la médecine de famille a aussi été organisée pour la première fois en 2016 dans une nouvelle configuration, sous forme de manifestation publique, à Berne.

\section{Stratégie 2018-2021}

Le Comité et les délégués de mfe œuvrent activement à la stratégie de l'association 2018-2021. Les objectifs principaux et les sous-objectifs ont déjà été définis conjointement; les mesures pour la mise en œuvre de ces objectifs sont actuellement élaborées et préparées en vue d'une adoption lors de l'assemblée des délégués d'automne.

\section{Assemblée générale 2018}

La prochaine assemblée générale se déroulera à nouveau dans le cadre du congrès du CMPR à Lucerne. Date: 21 juin 2018. 